\title{
The 'other' coral symbiont: Ostreobium diversity and distribution
}

\author{
Javier del Campo ${ }^{1}$, Jean-François Pombert ${ }^{2}$, Jan Šlapeta ${ }^{3}$, Anthony Larkum ${ }^{4}$ and Patrick J Keeling ${ }^{1}$ \\ ${ }^{1}$ Department of Botany, Canadian Institute for Advanced Research, University of British Columbia, \\ Vancouver, BC, Canada; ${ }^{2}$ College of Science, Illinois Institute of Technology, Chicago, IL, USA; ${ }^{3}$ The School of \\ Life and Environmental Sciences, Faculty of Veterinary Science, The University of Sydney, Sydney, NSW, \\ Australia and ${ }^{4}$ University of Technology Sydney, Ultimo NSW, Australia
}

\begin{abstract}
Ostreobium is an endolithic algal genus thought to be an early-diverging lineage of the Bryopsidales (Ulvophyceae, Chlorophyta). Ostreobium can live in low-light conditions on calcium carbonate substrata in tropical conditions. It is best known as a symbiont of corals, where it lives deep within the animal skeleton and exchanges nitrogen and carbon, as well as providing nutrients and photoassimilates. In contrast to the relatively well-studied role of the photosynthetic zooxanthellae symbionts in coral (Symbiodinium), Ostreobium phylogeny, diversity and distribution are all poorly understood. Here, we describe the phylogenetic position and diversity of Ostreobium based on plastid 16S ribosomal DNA (rDNA), 18S rDNA and rbcL genes from a nuclear genome survey and complete plastid genome, and determined its environmental diversity and distribution by screening the publicly available environmental data for those genes. The results shed light on the phylogeny and the ecology of the 'other' coral symbiont.
\end{abstract}

The ISME Journal (2017) 11, 296-299; doi:10.1038/ismej.2016.101; published online 15 July 2016

Coral reefs are important biodiversity hotspots, they protect our shores from erosion, mitigate the effect of the sea-level rise and are economically relevant for fisheries and tourism (Bellwood et al., 2004). Accordingly, there have been substantial efforts to preserve these ecosystems and protect the main and most threatened member of the consortia, the corals themselves. Corals are threatened by a number of factors associated with climate change, such as increase in sea temperature, sea-level rise, ocean acidification and the emergence of diseases (Bellwood et al., 2004). Of these, the most direct threat associated with an increase in the sea temperature is coral bleaching (Glynn, 1993). During bleaching events corals expel their zooxanthellae, which are symbionts of the dinoflagellate genus Symbiodinium that provide food to the animal in the form of photosynthates (Glynn, 1993). Because of the importance of this symbiotic association, Symbiodinium is by far the most-studied microorganism associated with corals, but is not the only coral symbiont. Indeed, the holobiont, the host coral and all its associated symbionts (Rosenberg et al., 2007), is much more complex, and includes a myriad of

Correspondence: J del Campo, Department of Botany, Canadian Institute for Advanced Research, University of British Columbia, 3529-6270 University Boulevard, Vancouver, British Columbia V6T1Z4, Canada.

E-mail: javier.delcampo@botany.ubc.ca

Received 21 May 2016; accepted 7 June 2016; published online 15 July 2016 bacteria (Rohwer et al., 2002), viruses (Wegley et al., 2007) and a diverse but understudied protist community. Among these protists there are both heterotrophs such as labyrinthulids (Harel et al., 2008; Siboni et al., 2010) or ciliates (Ulstrup et al., 2007; Lobban et al., 2011), a variety of apicomplexan-related lineages, some of which are photosynthetic (for example, Chromera) and some of which remain mostly uncharacterized (Janouškovec et al., 2012; Šlapeta and Linares, 2013), and lastly, the clearly photosynthetic genus, Ostreobium (Lukas, 1974).

Ostreobium is an endolithic alga that has been found to be associated with calcium carbonate structures in shallow tropical waters, including coral skeletons (Kornmann and Sahling, 1980). Ostreobium lives within the skeleton itself, sometimes at concentration so high that its visible as a green ring just below the living cnidarian (Supplementary Figure 1). It has been debated whether Ostreobium is a beneficial symbiont or parasite of the coral (Verbruggen and Tribollet, 2011), but the former seems more likely. It absorbs a wavelength of light not used by Symbiodinium (Fork and Larkum, 1989) and has been demonstrated to provide photosynthates to the coral (Schlichter et al., 1995). Ostreobium has even been proposed to replace zooxanthellae function during bleaching events, perhaps keeping the coral temporarily alive for a possible return of Symbiodinium (Fine and Loya, 2002). Despite the potential importance of Ostreobium in this system, little is known about the genus (Gutner-Hoch and Fine, 2011). 
Since even its phylogenetic position is uncertain, we first confirmed the relationship between Ostreobium and other ulvophytes based on three different markers derived from a nuclear genome survey and complete plastid genome (Supplementary Figure 2): plastid 16S rRNA gene, rbcL and nuclear 18S rRNA gene. The plastid 16S rRNA gene was the best represented in public databases and best resolved tree (Figure 1a), but the same result was found for $r b c L$ and $18 \mathrm{~S}$ rRNA gene (Supplementary Figure 3 and 4 and Supplementary Tables 2 and 3). The affiliation of Ostreobium within the Bryopsidales
(Verbruggen et al., 2009) was confirmed by all the three phylogenetic gene trees, but only statistically supported by $16 \mathrm{~S}$ rRNA gene (with $100 \%$ bootstrap support). Because plastids are sampled along with bacteria in microbial diversity surveys, the 16S rRNA gene tree also included a significant number of environmental Ostreobium sequences (ten times more than rbcL or 18S rRNA gene: Supplementary Table 1). In the plastid 16S tree, these sequences formed a strongly supported monophyletic group, but were subdivided into three distinct clades (1,2 and 3), all with 100\% support

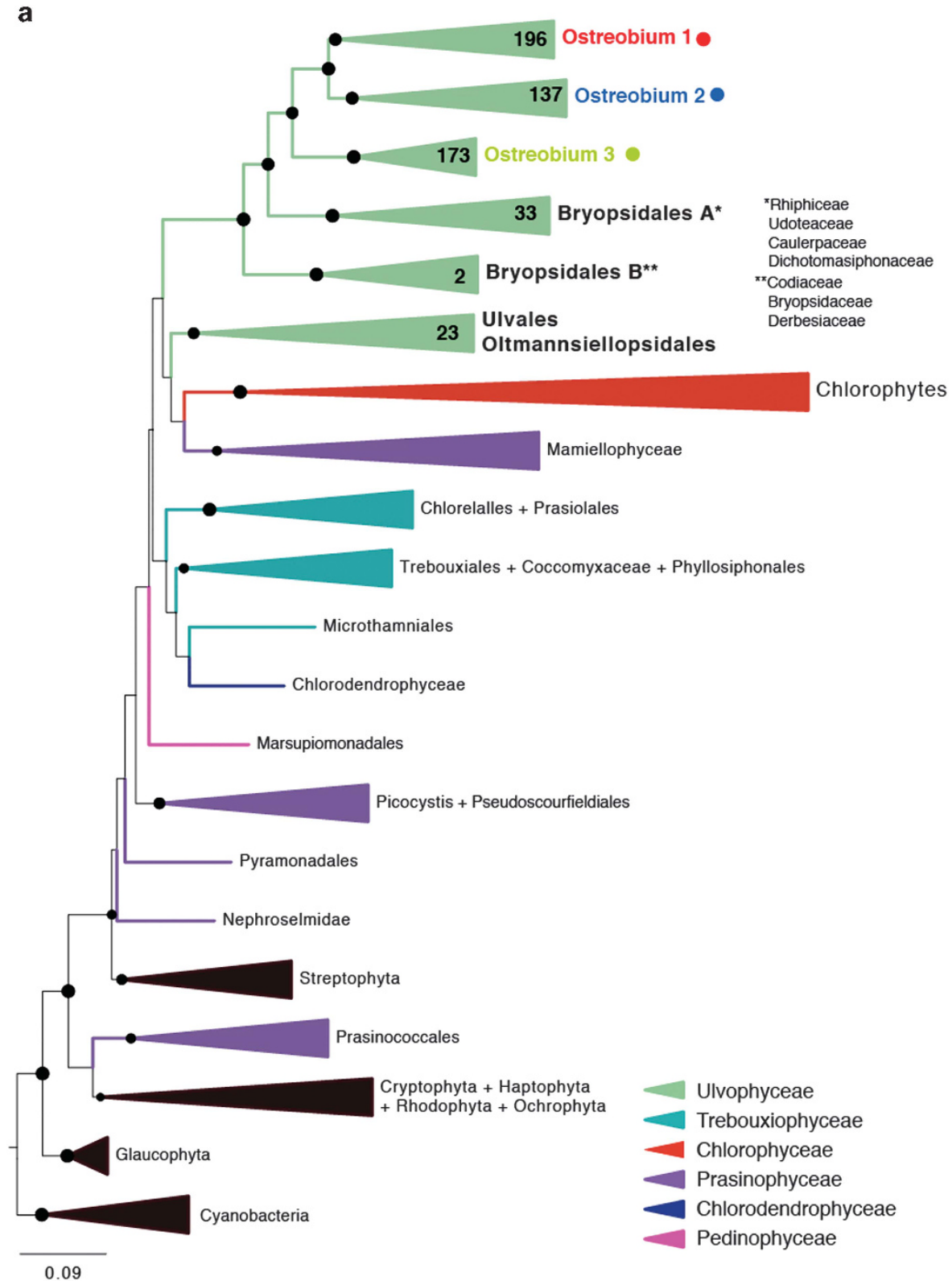

Figure 1 (a) Maximum likelihood phylogenetic tree inferred from the plastid 16S rRNA gene. Taxa includes clustered sequences $(97 \%)$ from a curated database of ulvophytes $(n=89$, representing 590), a selection of other green algae $(n=41)$ and an outgroup of non-chlorophytes $(n=27)$. The three Ostreobium clades are labeled at the top, and color-coded consistently throughout the figure. (b) Biogeographical distribution of Ostreobium clades based on the 16S rRNA gene metadata. (c) Host distribution of Ostreobium clades based on the 16S rRNA gene sequence metadata and the host geographical origin. Clade 1 is widespread but dominates in Acroporidae, clade 3 is absent from complex corals and only clade 2 was found in octocorals. 


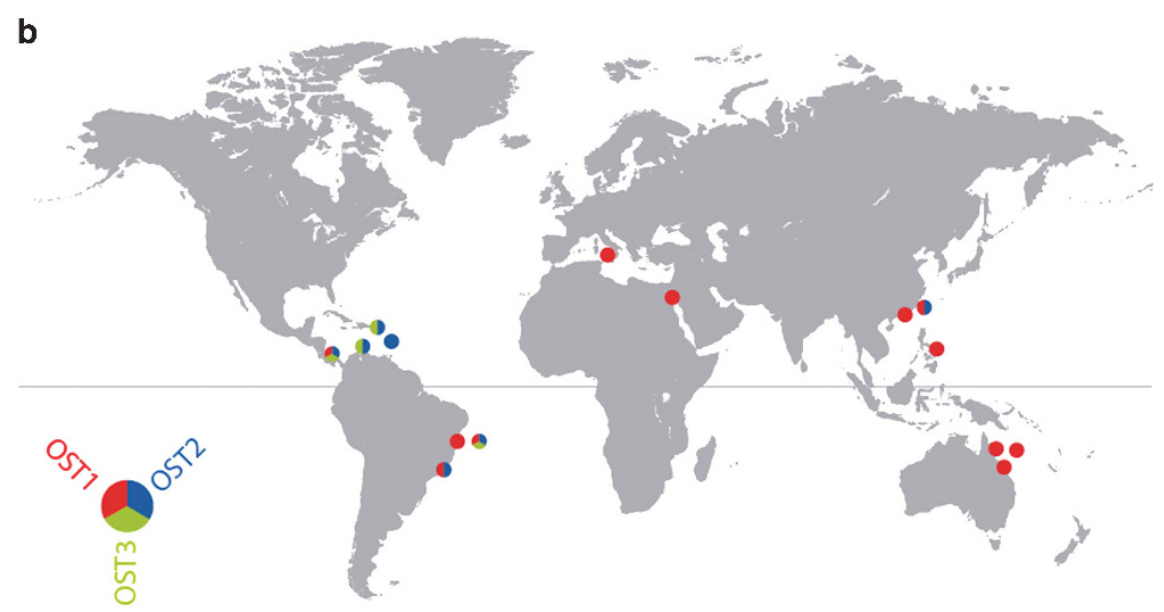

c

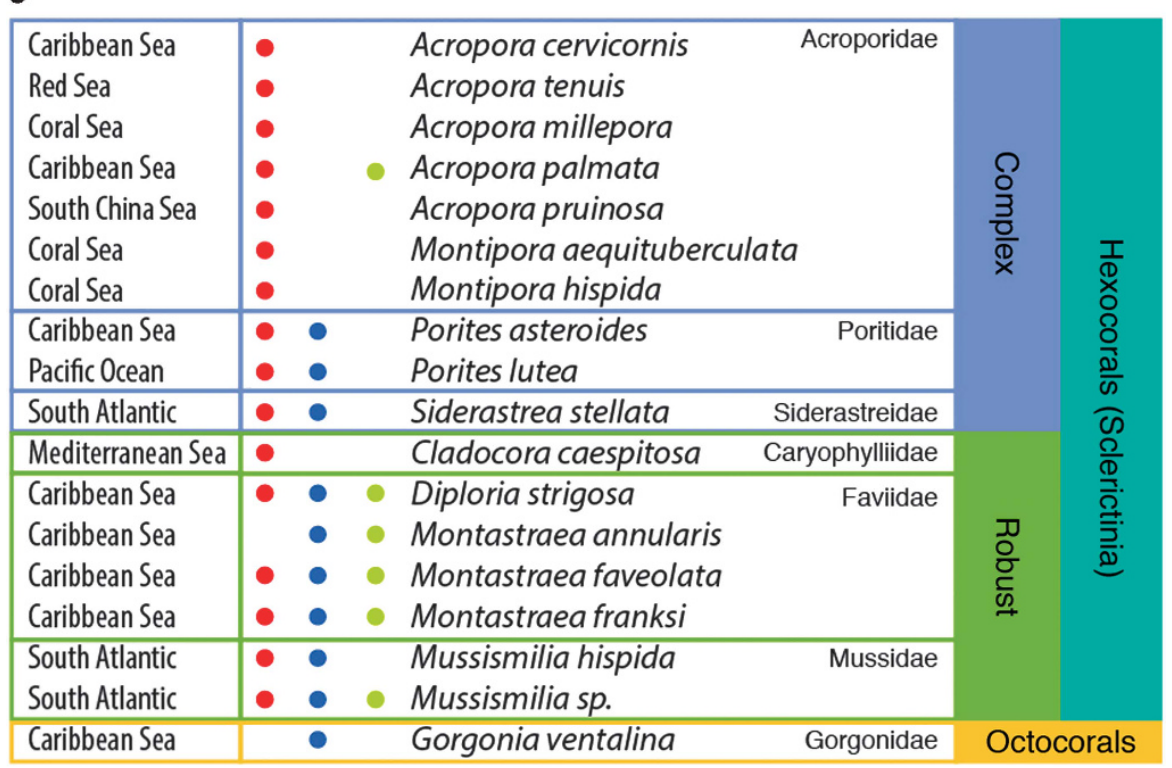

Figure 1 Continued.

(Figure 1a). The sequence level diversity within these groups is similar to the rest of the Bryopsidales combined, and the divergence between any two subgroups is typically greater than that between bryopdisalean genera, altogether suggesting that a substantial amount of diversity exists within what we call 'Ostreobium'.

The environmental 16S rRNA gene data also presents the first opportunity to study the geographical distribution of Ostreobium (and indeed is currently the only molecular marker with sufficient data to address such questions: only a few $r b c L$ genes are known and all are from strains that fall into the same clade as our genomic isolate, and the genomic isolate provided the first and only nuclear $18 \mathrm{~S}$ from this genus). We obtained environmental metadata from all the publicly available Ulvophyte environmental sequences in order to link these sequences to their geographical distribution, environment and, eventually, host (Supplementary Table 1). This immediately revealed that Ostreobium is almost exclusively associated with coral reefs: we found no association with shells as it has previously been described (Kornmann and Sahling, 1980), and the two sequences that are not associated to corals were obtained from reef-dwelling sponges. Even more interesting, however, is that the three well-supported Ostreobium clades show a complex biogeographical distributed across different reefs (Figure 1b). Ostreobium clade 1 is most common globally, but is relatively rare in the Caribbean. In contrast, clades 2 and 3 are more common in the Caribbean, present in the South Atlantic, and rare elsewhere. Interestingly, the distribution of Ostreobium clade 1 overlaps with Symbiodinium C-dominated regions, whereas Ostreobium clade 2 and 3 overlap with Symbiodinium A and B-dominated regions. Of course, these distribution patterns represent dominance and not simple presence and absence, and other local aspects of distribution (such as depth, latitude, and host-associations) make the patterns more complex for Symbiodinium (Baker, 2003) and probably also Ostreobium. But the apparent degree 
of overlap in biogeographical distributions between these two unrelated symbionts suggests a possible correlation, which would require detailed analysis based on additional data specifically testing the detailed distribution of Ostreobium in nature. Finally, we investigated the distribution of Ostreobium clades among coral host species (Figure 1c), and observed Ostreobium clade 1 to dominate in Acroporidae, and Ostreobium clade 3 to be virtually absent from complex corals. Only Ostreobium clade 2 was found in octocorals, but from a single data set. The number of analyzed host in the different areas is limited so there is the possibility that this distribution might be affected by the general biogeographic patterns.

Our observations regarding the environmental diversity and distribution of Ostreobium indicate first of all that Ostreobium is closely associated to corals and not widespread in other environment that has been sampled. Second, the distribution of Ostreobium suggests that it shares similar biogeographical patterns as Symbiodinium, and that distinct subclasses have host species preference. We hypothesize that Ostreobium might have co-evolved with both coral and Symbiodinium, being a stable member of the holobiont through the ages. Further analyses are needed to improve our knowledge about the biogeographical and host distribution of Ostreobium if we are to test this, and to determine the nature of the relationship of its relationship with Symbiodinium.

\section{Conflict of Interest}

The authors declare no conflict of interest.

\section{Acknowledgements}

This work was supported by a grant from the Natural Sciences and Engineering Research Council of Canada (227301) and from a grant to the Centre for Microbial Diversity and Evolution from the Tula Foundation. PJK is a Senior Fellow of the Canadian Institute for Advanced Research. JdC was supported by a Marie Curie International Outgoing Fellowship grant (FP7-PEOPLE-2012-IOF - 331450 CAARL).

\section{References}

Baker AC. (2003). Flexibility and specificity in coral-algal symbiosis: diversity, ecology, and biogeography of Symbiodinium. Annu Rev Ecol Evol Syst 34: 661-689.

Bellwood DR, Hughes TP, Folke C, Nyström M. (2004). Confronting the coral reef crisis. Nature 429: 827-833.

Fine M, Loya Y. (2002). Endolithic algae: an alternative source of photoassimilates during coral bleaching. Proc R Soc B Biol Sci 269: 1205-1210.
Fork D, Larkum A. (1989). Light harvesting in the green alga Ostreobium sp., a coral symbiont adapted to extreme shade. Mar Biol 103: 381-385.

Glynn PW. (1993). Coral reef bleaching: ecological perspectives. Coral Reefs 12: 1-17.

Gutner-Hoch E, Fine M. (2011). Genotypic diversity and distribution of Ostreobium quekettii within scleractinian corals. Coral Reefs 30: 643-650.

Harel M, Ben-Dov E, Rasoulouniriana D, Siboni N, Kramarsky-Winter E, Loya Y et al. (2008). A new Thraustochytrid, strain Fng1, isolated from the surface mucus of the hermatypic coral Fungia granulosa. FEMS Microbiol Ecol 64: 378-387.

Janouškovec J, Horák A, Barott KL, Rohwer FL, Keeling PJ. (2012). Global analysis of plastid diversity reveals new apicomplexan-related lineages associated with coral reefs. Curr Biol 22: R518-R519.

Kornmann P, Sahling P. (1980). Ostreobium quekettii (Codiales, Chlorophyta). Helgoländer Meeresuntersuchungen 122: 115-122.

Lobban CS, Raymundo LM, Montagnes DJS. (2011). Porpostoma guamensis n. sp., a philasterine scuticociliate associated with brown-band disease of corals. J Eukaryot Microbiol 58: 103-113.

Lukas K. (1974). Two species of the chlorophyte genus Ostreobium from skeletons of Atlantic and Caribbean Reef Corals. J Phycol 10: 331-335.

Rohwer FL, Seguritan V, Azam F, Knowlton N. (2002). Diversity and distribution of coral-associated bacteria. Mar Ecol Prog Ser 243: 1-10.

Rosenberg E, Koren O, Reshef L, Efrony R, Zilber-Rosenberg I. (2007). The role of microorganisms in coral health, disease and evolution. Nat Rev Microbiol 5: 355-362.

Schlichter D, Zscharnack B, Krisch H. (1995). Transfer of photoassimilates from endolithic algae to coral tissue. Naturwissenschaften 82: 561-564.

Šlapeta J, Linares MC. (2013). Combined amplicon pyrosequencing assays reveal presence of the apicomplexan 'type-N' (cf. Gemmocystis cylindrus) and Chromera velia on the Great Barrier Reef, Australia. PLOS ONE 8: e76095.

Siboni N, Rasoulouniriana D, Ben-Dov E, KramarskyWinter E, Sivan A, Loya Y et al. (2010). Stramenopile microorganisms associated with the massive coral Favia sp. J Eukaryot Microbiol 57: 236-244.

Ulstrup KE, Kühl M, Bourne DG. (2007). Zooxanthellae harvested by ciliates associated with brown band syndrome of corals remain photosynthetically competent. Appl Environ Microbiol 73: 1968-1975.

Verbruggen $\mathrm{H}$, Ashworth M, LoDuca ST, Vlaeminck C, Cocquyt E, Sauvage T et al. (2009). A multi-locus time-calibrated phylogeny of the siphonous green algae. Mol Phylogenet Evol 50: 642-653.

Verbruggen H, Tribollet A. (2011). Boring algae. Curr Biol 21: R876-R877.

Wegley L, Edwards R, Rodriguez-Brito B, Liu H, Rohwer F. (2007). Metagenomic analysis of the microbial community associated with the coral Porites astreoides. Environ Microbiol 9: 2707-2719.

Supplementary Information accompanies this paper on The ISME Journal website (http://www.nature.com/ismej) 